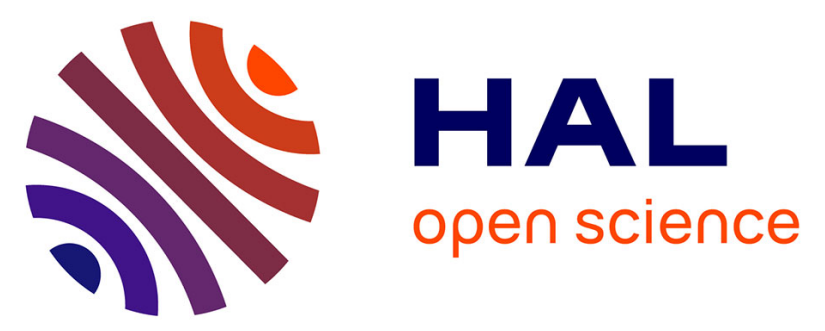

\title{
Energy Tuning of Electronic Spin Coherent Evolution in Methylammonium Lead Iodide Perovskites
}

\author{
Guadalupe Garcia-Arellano, Gaëlle Trippé-Allard, Laurent Legrand, Thierry \\ Barisien, Damien Garrot, Emmanuelle Deleporte, Frédérick Bernardot, \\ Christophe Testelin, Maria Chamarro
}

\section{To cite this version:}

Guadalupe Garcia-Arellano, Gaëlle Trippé-Allard, Laurent Legrand, Thierry Barisien, Damien Garrot, et al.. Energy Tuning of Electronic Spin Coherent Evolution in Methylammonium Lead Iodide Perovskites. Journal of Physical Chemistry Letters, 2021, 12 (34), pp.8272-8279. 10.1021/acs.jpclett.1c01790 . hal-03370543

\section{HAL Id: hal-03370543 \\ https://hal.science/hal-03370543}

Submitted on 8 Nov 2021

HAL is a multi-disciplinary open access archive for the deposit and dissemination of scientific research documents, whether they are published or not. The documents may come from teaching and research institutions in France or abroad, or from public or private research centers.
L'archive ouverte pluridisciplinaire HAL, est destinée au dépôt et à la diffusion de documents scientifiques de niveau recherche, publiés ou non, émanant des établissements d'enseignement et de recherche français ou étrangers, des laboratoires publics ou privés. 


\title{
Energy tuning of the Electronic Spin Coherent
}

\section{evolution in Methylammonium Lead Iodide}

\section{Perovskites}

*Guadalupe Garcia-Arellano', Gaëlle Trippé-Allard', Laurent Legrand', Thierry Barisien', Damien Garrot", Emmanuelle Deleporte', Frédérick Bernardot', Christophe Testelin' and Maria Chamarro'

'Sorbonne Université, CNRS, Institut des NanoSciences de Paris, 4 place Jussieu, F-75005 Paris, France

${ }^{2}$ Université Paris-Saclay, ENS Paris-Saclay, Centrale Supélec, CNRS, LuMIn, F-91190 Gif-surYvette, France

¿Université Paris-Saclay, UVSQ, CNRS, GEMaC, F-78000 Versailles, France.

*Email: arellano@insp.jussieu.fr

\begin{abstract}
We investigated the coherent evolution of the electronic spin at low temperature in high-quality $\mathrm{CH}_{3} \mathrm{NH}_{3} \mathrm{~Pb} \mathrm{I}_{3}$ polycrystalline films, by picosecond-resolved photo-induced Faraday rotation. We evidenced that this coherent evolution can be tuned by choosing the pump-probe energy within the lowest optical-absorption band, and we explained it as the result of two main contributions: the localized electron and the localized hole. Their corresponding amplitudes ratio is not constant across the lowest absorption band -an observation which disqualifies a free exciton from being at the origin of the electronic spin coherent evolution. We measured a spin coherence time of localized electrons (holes) of $4.4 \mathrm{~ns}(3.7 \mathrm{~ns})$ at $1.635 \mathrm{eV}$, which evolves to about $7 \mathrm{~ns}$ at $1.612 \mathrm{eV}$ (the hole coherence time remains almost constant at lower energies). Finally, we give a global
\end{abstract}


image of the spin coherent evolution in bulk metal halide perovskite, which overcomes recent controversies.

\section{TOC GRAPHICS}

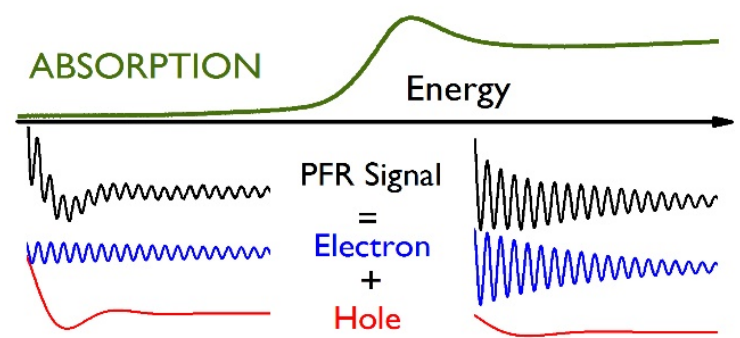

KEYWORDS: hybrid-halide perovskites, MAPI, spin dynamics, spin relaxation time, Photoinduced Faraday rotation.

Hybrid metal-halide perovskites such as Methylammonium lead iodide $\mathrm{CH}_{3} \mathrm{NH}_{3} \mathrm{PbI}_{3}$ (MAPI) are the center of a huge quantity of studies, due to their outstanding optoelectronic properties and the possibilities opened in the photo-voltaic field. Clear signatures of excitons have been evidenced in low-temperature magneto-absorption experiments of this material [1] and other perovskite materials [2-4], with the consequent determination of the exciton binding energy and its effective mass. As more conventional high-purity bulk semiconductors [5-7], the photoluminescence spectra (PL) seem to be dominated by defects or impurity related luminescence [8-11]. First-principle calculations have demonstrated a particular physics of defects in MAPI, characterized by comparable formation energies for dominant donor and acceptor defects, and the presence of shallow energy levels for dominant intrinsic defects $[12,13]$.

Hybrid halide perovskites are highly promising materials also due to their large and tunable spinorbit coupling, spin-dependent optical selection rules, and their predicted electrically tunable Rashba spin splitting [14-15]. Due to the presence of heavy atoms ( $\mathrm{Pb}, \mathrm{I})$, the spin-orbit coupling is very important in lead perovskite materials and makes then possible the optical generation of electronic spins. The counterpart of this important spin-orbit coupling is that spin relaxation and dephasing mechanisms are enhanced; the created electronic spins could then relax in a very fast manner. Recently, spin relaxation times of the order of ns have been observed at low temperature in bulk MAPI polycrystalline films [16] and also in bulk $\mathrm{CsPBr}_{3}$ crystals [17]. At higher 
temperatures, the spin relaxation time becomes shorter, in the picosecond range [16,18-20]. Odenthal and coworkers, in Ref. [16], have interpreted their observations at low temperature as resulting from a quantum beating associated to the fine exciton structure in orthorhombic material; meanwhile, in Ref. [17], Belykh et al. interpreted their results as the signature of localized electrons and holes at separated spatial areas. The relative role of free or localized carriers and free or localized excitons is a key question in a large variety of optical effects and spin dynamic processes in semiconductor materials. That is why, we are interested here to give a global understanding of the spin dynamics in bulk perovskite materials at low temperature.

In this work, we have measured in MAPI polycrystalline films the transverse spin coherence time by means of a photo-induced Faraday rotation technique (PFR) [21]. Thanks to the picosecond resolution of the experimental setup, we can select different excitation energies with a band-width of about $1 \mathrm{meV}$. We have found that is possible to tune, in this way, the coherent electronic spin evolution signal by exciting at different energies. We demonstrate the optical orientation of localized electrons and holes in this perovskite material at low temperature by different analyses and discussions.

First, to characterize the optical transition energies, we have measured the absorption spectra of MAPI samples as a function of temperature. The evolution of the absorption peak at low energy is consistent with the orthorhombic-tetragonal transition as shown by other authors [22] (see Figure S1(a) in SI). Figure 1 (a) shows the absorption spectrum measured at $10 \mathrm{~K}$. The PFR measurements being performed at $2 \mathrm{~K}$, we have also drawn the estimated absorption spectrum at $2 \mathrm{~K}$, using the extrapolation of the energy position of the absorption peak (see Figure S1(b) in the SI). We tuned the pump-probe energy within the lowest absorption band of MAPI centered at $1.635 \mathrm{eV}$ (see Figure 1(a)). Figure 1(b) shows the time-evolution of the PFR signal as the pumpprobe energy changes from $1.612 \mathrm{eV}$ to $1.646 \mathrm{eV}$, when a transverse magnetic field $\mathrm{B}=200 \mathrm{mT}$ is applied in Voigt geometry, at $\mathrm{T}=2 \mathrm{~K}$. Two components are revealed by the Fast Fourier Transform (FFT) of the PFR signal (see Fig. 1(c)), in agreement with recent results in a similar MAPI sample [16]: the low-frequency amplitude is noted $A_{h}$ and the high-frequency one $A_{e}$. 

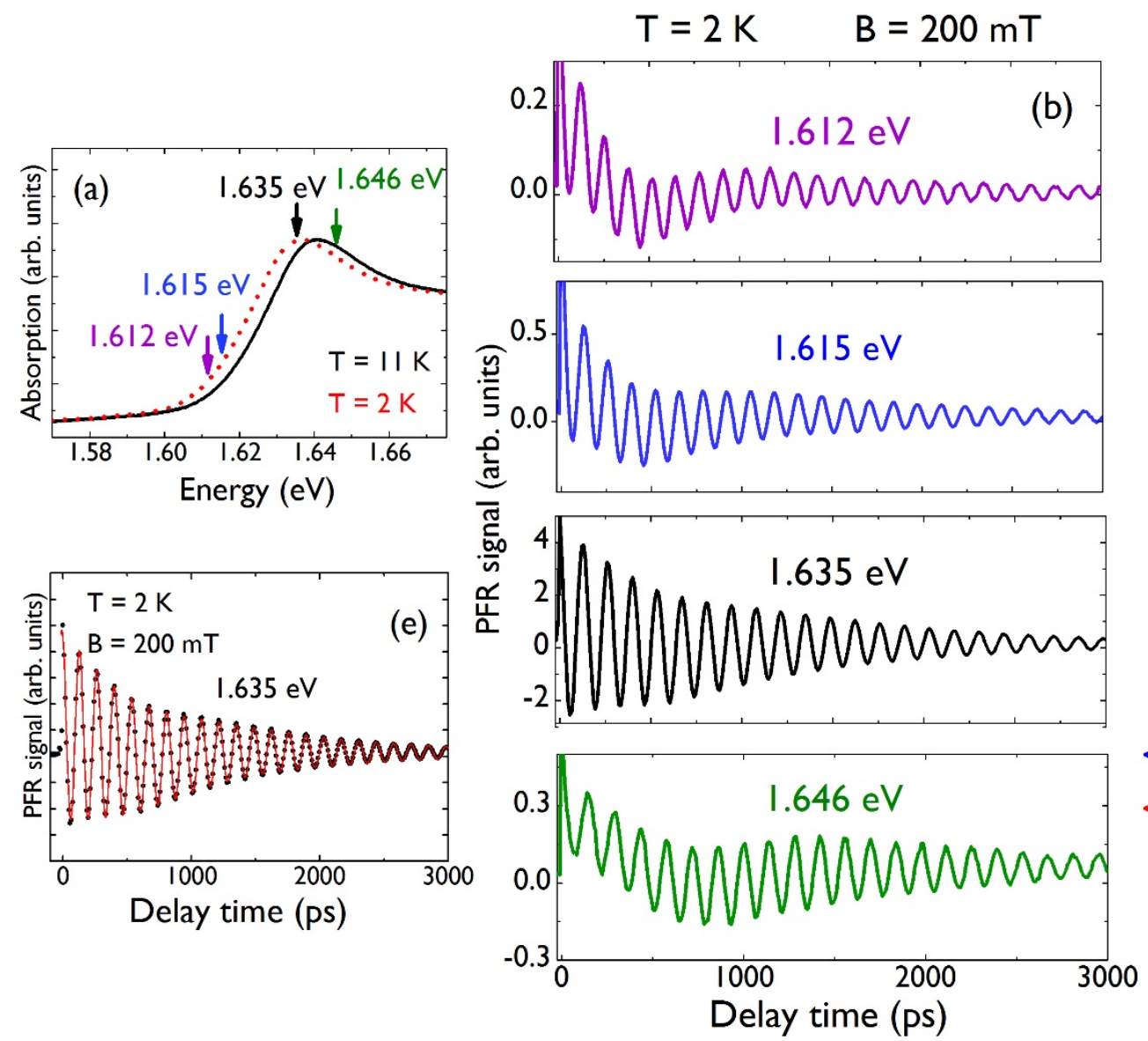
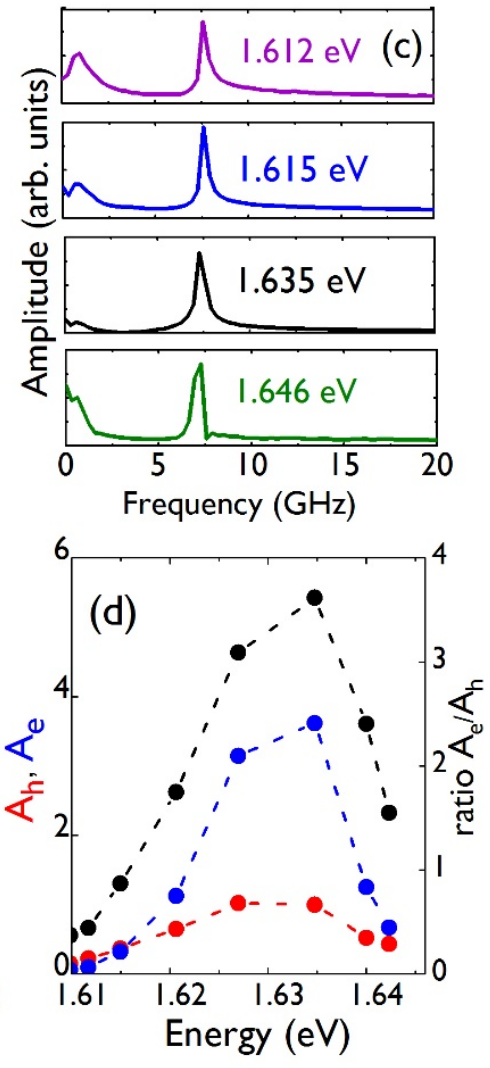

Fig. 1: (a) Absorption spectra obtained on MAPI sample at $\mathrm{T}=11 \mathrm{~K}$ (solid black line), and $\mathrm{T}=2$ $\mathrm{K}$ (red dotted line) calculated by extrapolation (see section I of the SI); the arrows in the spectra indicate the excitation energies chosen to perform PFR experiments. (b) PFR signals obtained on MAPI sample at a fixed magnetic field $\mathrm{B}=200 \mathrm{mT}$ and $\mathrm{T}=2 \mathrm{~K}$, at different energies. (c) FFT of the corresponding PFR signals. (d) Evolution of the amplitudes vs energy of the two contributions extracted from the fit of PFR signal at different energies with Eq. (1) or Eq. (4). (e) PFR signal obtained at $\mathrm{E}=1.635 \mathrm{eV}$ (black points) and fit (red solid line) of the Eq. (1) to the experimental data.

The PFR signal near the maximum of the absorption peak is well described by the expression:

$$
\theta_{F}=A_{e} e^{-t / T_{2, e}^{*}} \cos \left(2 \pi f_{e} t+\varphi_{e}\right)+A_{h} e^{-t / T_{2, h}^{*}} \cos \left(2 \pi f_{h} t+\varphi_{h}\right),
$$

where $f_{e}, f_{h}$ are the two oscillation frequencies identified in the FFT, $T_{2, e}^{*}, T_{2, h}^{*}$ the corresponding dephasing times at the given magnetic field, and $\varphi_{e}$ and $\varphi_{h}$ the initial phases. Figure 1(e) shows 
the fit of the PFR signal at $\mathrm{B}=200 \mathrm{mT}$ and $1.635 \mathrm{eV}$ using Eq. (1). The PFR signal is also given in Figure 1(b) for different pump-probe energies. At the lowest energy, we need a third component with a very short dephasing time and a frequency comparable to $f_{e}$ (this new frequency cannot be defined with precision because of the short dephasing time of this component). The energy dependence of the ratio $A_{e} / A_{h}$ obtained from the fitting of the PFR signals at different energies is presented in Fig. 1(d). As we can see, both contributions, $A_{e}$ and $A_{h}$, are maximal near the absorption maximum and the amplitude ratio $A_{e} / A_{h}$ depends on the pump-probe energy, being maximum at $1.635 \mathrm{eV}$.

In Ref. [16], the authors interpreted the measured coherent evolution as the result of exciton quantum beats, with two frequencies associated to the hole and the electron, respectively. For an free exciton, the ratio $A_{e} / A_{h}$ should remain constant across the lowest absorption peak. Here, we clearly demonstrate that this is not the case.

Even if the existence of exciton quantum beats is theoretically possible due to the exciton fine structure of MAPI orthorhombic materials [23-27] its observation depends on different parameters and is generally an exceptional event. In orthorhombic perovskite materials, four energetically closely spaced transitions can be excited by a circularly polarized short pulse, and after excitation, the induced polarizations in the medium oscillate with slightly different frequencies. Their interferences could be at the origin of exciton quantum beats (see section 3 in SI). To be able to observe exciton quantum beatings, at least two conditions are necessary: a resonant excitation and a lifetime and/or a coherence time of free excitons larger than the beating period. The resonant excitation can be accomplished in PFR measurements but the photoluminescence of MAPI is clearly shifted to lower energies with respect to the maximum of the absorption spectrum (see Fig. S1 (c) in SI); that is an indication that the photoluminescence is dominated by defects or impurities [8-11] and it is also a signature of the fast relaxation of free excitons to localized centers. In this sense, MAPI polycrystalline films behave similarly to other more conventional direct-gap semiconductors like GaAs [28,29] or CdTe [30] with smaller spinorbit coupling. The latter materials contain p- or n-type impurities even when they are fabricated to obtain very high-quality materials by molecular beam epitaxy. The excitons created in semiconductor bulk materials in a resonant or quasi-resonant excitation relax very fast to localized centers. 
Figures 2(a) and (b) show the 2K PFR signal measured in the sample for different values of the transverse magnetic field (Voigt geometry) at the maximum $(1.635 \mathrm{eV})$ and at the low-energy side $(1.612 \mathrm{eV})$ of the lowest absorption band. PFR measurements were fitted by Eq. (1). When the excitation energy is shifted to $1.612 \mathrm{eV}$, the hole contribution with respect to the electron one increases. For the amplitudes extracted from the fit, see section 4 in the SI. The corresponding FFT have been also represented in the SI (see Fig. S2). Figures 2(c) and (d), upper panels, show the dependence of the two extracted angular frequencies $\omega_{i}=2 \pi f_{i}(i=\mathrm{e}, \mathrm{h})$ on the applied magnetic field $B$ at $1.635 \mathrm{eV}$ and $1.612 \mathrm{eV}$, respectively. The two frequencies increase linearly with the magnetic field for both energies. From the slopes of the two linear fits, we obtained two Landé factors using the relation:

$$
\omega_{i}=\frac{\left|g_{i}^{\perp}\right| \mu_{B}}{\hbar} B
$$

where $\mu_{B}$ is the Bohr magneton, $\hbar$ the reduced Planck constant, and $g_{i}^{\perp}$ is the transverse Landé factor of the corresponding species. At $1.635 \mathrm{eV}$, we obtain the two Landé factors $\left|g_{h}^{\perp}\right|=$ $0.238 \pm 0.002$ and $\left|g_{e}^{\perp}\right|=2.593 \pm 0.006$, in agreement with the Yu calculations [31], and both Landé factors are larger at $1.612 \mathrm{eV}$. The observed linear dependence of the beating frequencies on the magnetic field leads us to conclude, as we discuss below, that in the PFR signal, we do not observe beating of the exciton as previously observed in quantum wells of more conventional semiconductors (GaAs, $\mathrm{CdTe})$. 

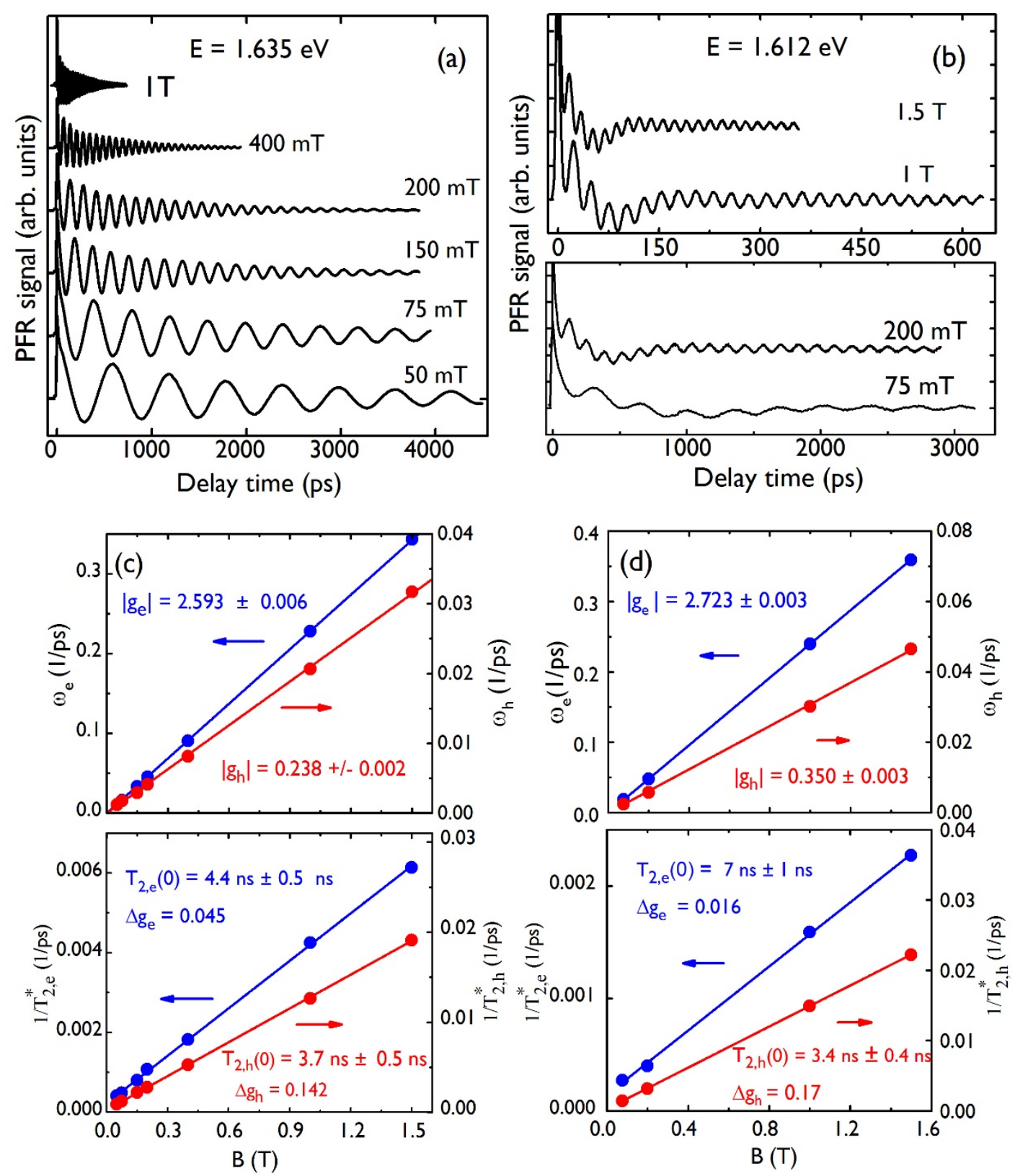

Fig. 2. PFR signals for MAPI sample as a function of the pump-probe delay, obtained at $\mathrm{T}=2 \mathrm{~K}$ for several values of a transversal magnetic field, working at the peak energy of the $2 \mathrm{~K}$ absorption spectrum: (a) $\mathrm{E}=1.635 \mathrm{eV}$; and at a lower energy: (b) $\mathrm{E}=1.612 \mathrm{eV}$.

(c) and (d), top figures: Experimental data for the Larmor frequency of the electron (blue disks) and hole (red disks) for the two studied energies, and linear fits (solid lines) with Eq. (2). (c) and (d), bottom figures: spin dephasing rates for the electron (blue disks) and hole (red disks) and linear fits (solid lines) with Eq. (3). 
The exciton beatings in a transverse magnetic field, in presence of a fine structure splitting (FSS), were first observed in GaAs quantum wells [32,33], because the electronic confinement makes easier the observation of the exciton in photoluminescence spectra and enhances the electron-hole exchange interaction leading to larger FSS than in the bulk. Later on, exciton quantum beats were also observed in quantum dots [34-37]. The frequency of the exciton spin beatings obtained under resonant excitation clearly showed a non-linear dependence on magnetic field when the FSS is larger or comparable to the Zeeman splitting [32]. In zinc-blende semiconductors, the electron-hole exchange interaction split heavy-hole exciton states into two dark (showing no allowed optical transitions) and two bright states (at the origin of allowed optical transitions) separated by the energy $\hbar \omega_{0}$. In an applied transverse magnetic field, the exciton quasi-stationary states are linear combination of dark and bright excitons. A circularly polarized resonant pump will generate a superposition of the two eigenstates and a beating of the exciton at a frequency $\sqrt{\omega_{0}^{2}+\omega_{Z}^{2}}$, with $\hbar \omega_{Z}=g_{X} \mu_{B} B$ ( $g_{X}$ is the exciton Landé factor), and the magnetic-field-independent $\omega_{0}$. In GaAs quantum wells, the spin coherence time observed was of the order of several tens of ps and the bright-dark splitting $\hbar \omega_{0}$ of the order of $100 \mu \mathrm{eV}$.

For lead halide perovskites, the magnetic field dependence of the beating exciton frequencies is more complex. At the band edge, the photo-created electron $\left|J=\frac{1}{2} ; J_{z}= \pm \frac{1}{2}\right\rangle$ and hole $\left|S=\frac{1}{2} ; S_{z}= \pm \frac{1}{2}\right\rangle$ lead to four excitonic states split into a dark singlet state $\left|O_{D}\right\rangle$ (optically inactive) and a bright triplet state $\left(\left|O_{B}\right\rangle,| \pm 1\rangle\right)$ (optically active). The triplet degeneracy is partially lifted in the tetragonal phase, or totally lifted in the orthorhombic phase, via the crystal field contributions [24,25] (see also section 3 in SI). In the orthorhombic phase, three bright states are coupled to linearly polarized light and noted $|X\rangle,|Y\rangle$ and $|Z\rangle$. The FSS is enlarged in confined nanostructures such as nanocrystals. The FSS has then been experimentally evidenced firstly in inorganic $\mathrm{CsPbBr}_{3}-\mathrm{Cl}_{*}$ nanocrystals [23, 38-39], then in hybrid organic-inorganic $\mathrm{FAPbBr}_{3}$ nanocrystals, made with formamidinium (FA) [40], and more recently in $\mathrm{CsPbI}_{3}$ nanocrystals [41]. Very recently, the FSS has also been observed in bulk materials such as $\mathrm{MAPbBr}_{3}$, with a splitting of bright excitons equal to $200 \mu \mathrm{eV}$ [27]. All these experimental results have led to a better estimate of the FSS in bulk organic lead halide perovskites, either based on formamidinium or methylammonium [25]. The FSS is shown in figure S3-I in SI for the three crystal phases: cubic $\left(\mathrm{O}_{n}\right)$, tetragonal $\left(\mathrm{D}_{2 n}\right)$ and orthorhombic $\left(\mathrm{D}_{2 n}\right)$. The crystal phase of $\mathrm{MAPbI}_{3}$ at low temperature is supposed to be orthorhombic, and the splittings are estimated $\delta_{B D}=400 \mu \mathrm{eV}, \Delta E_{1}=95 \mu \mathrm{eV}$ and $\Delta E_{2}=205 \mu \mathrm{eV}$ (see section 3 of the SI). These values are 
almost two orders of magnitude larger than the splitting (few $\mu \mathrm{eV}$ ) considered in Ref. [16]. Taking into account the recent experimental findings in $\mathrm{MAPbBr}_{3}$ and recent calculations in $\mathrm{MAPbI}_{3}$, we have been able to predict the evolution of the exciton beating frequencies as a function of the magnetic field (given in section 3 in SI). We underline that we obtain four different frequencies that show a clear non-linear evolution versus magnetic field (see SI), not observed in our measurements. This expected excitonic behavior is in contradiction with the linear behavior of the experimentally observed frequencies with magnetic field.

Figures 2 (c) and (d), lower panels, show the spin dephasing rate $1 / T_{2, i}^{*}$ versus the applied magnetic field, obtained at the maximum and at the low-energy side of the absorption spectrum, $1.635 \mathrm{eV}$ and $1.612 \mathrm{eV}$ respectively. We underline that $T_{2, i}^{*}$ shortens with increasing $B$, due to the inhomogeneities of the g-factor values. The g-factor distribution width, $\Delta g_{i}$, and the spin dephasing time extrapolated at zero magnetic field $T_{2, i}(0)$ of a single electron or hole can be determined using the relation:

$$
\frac{1}{T_{2, i}^{*}}=\frac{1}{T_{2, i}(0)}+\frac{\Delta g_{i} \mu_{B}}{\hbar} B
$$

where $T_{2, i}(0)$ is also equal to the longitudinal electron or hole spin relaxation time at zero magnetic field. Figures 2 (c) and (d), lower panels, show that the extrapolation of the experimental data to zero magnetic field leads to the electron and hole spin relaxation times for both detection energies. The electron spin relaxation time increases from $T_{2, e}(0)=4.4 \mathrm{~ns}$ at the maximum of the lowest absorption band to $T_{2, e}(0)=7 \mathrm{~ns}$ at $1.612 \mathrm{eV}$; meanwhile we obtain a quasi-constant hole spin relaxation time of about $T_{2, h}(0)=3.5 \mathrm{~ns}$. We underline that, whatever the detection energy, the dispersion on the $\mathrm{g}_{\mathrm{e}}$ factor is much smaller than the dispersion on the $\mathrm{g}_{\mathrm{h}}$ factor. By combining information obtained from the fits in Figs. 2 (c) and (d), we also obtain at $1.635 \mathrm{eV}: \Delta g_{h} / g_{h}=60 \%$ and $\Delta g_{e} / g_{e}=1,7 \%$. At $1.612 \mathrm{eV}$, weaker dispersions, $\Delta g_{h} / g_{h}=$ $49 \%$ and $\Delta g_{e} / g_{e}=0,6 \%$, are measured.

When the excitons are optically oriented, they can preserve partially or completely their spin orientation during the localization process. Many of the most important findings on spin relaxation were obtained in n-doped GaAs thin layers. The study of the electronic spin relaxation at low temperature in the insulator regime was explained by the interplay of two main 
mechanisms whose importance depends on the electron concentration [29]: the hyperfine interaction [42], and the anisotropic exchange interaction [43]. It was demonstrated that the spin relaxation time can vary by more than three orders of magnitude depending on electron density and temperature. In GaAs, the maximum spin relaxation time was of the order of 1 microsecond at low temperature [44]. The measured spin relaxation times are quite long to be identified as the spin coherence time of exciton in MAPI, since this material has an important electron-hole exchange interaction (discussed above). However, the values are consistent with the localization hypothesis. In the future, as done in GaAs, more studies are needed to determine the relative importance of different spin relaxation mechanisms.

We have also obtained the magnetic-field dependencies of $A_{e}$ and $A_{h}$ (see Figure S4-III (b) of the $\mathrm{SI})$. At the maximum of absorption peak, $\mathrm{A}_{\mathrm{h}}$ is almost constant for magnetic fields smaller than 1.6 T. Meanwhile $\mathrm{A}_{\mathrm{e}}$ shows a maximum at low magnetic fields and slightly decreases with increasing magnetic fields. Odenthal's description of exciton beatings predicts the magneticfield dependence for the two observed components of frequencies (see Figure S8 in SI of Ref. [16]). Under this model, the amplitude of the electron component increases from zero at zero magnetic field to a cap value, and the amplitude of the hole component decreases from a maximum at zero magnetic field to a cap value similar to the cap value of the electron. We underline that our experimental observations do not follow the predicted magnetic-field dependence discussed in Ref. [16]. All the discussed evidences concerning the magnetic-field dependence of both frequencies and amplitudes lead us to conclude that in the PFR signal, we do not observe beatings of the exciton, as previously observed in conventional semiconductors (GaAs, CdTe).

As announced at the beginning, we propose that the two observed frequencies are related to the presence of localized electrons and holes. At low temperature by choosing an excitation energy in the lowest absorption peak (see Figure 1 a)) we create free excitons but this population of free excitons evolves very quickly towards a population of bound excitons due to the presence of defects $[9,11,22]$. These excited states recombine radiatively and non-radiatively and lead to localized electrons and holes. During all these relaxation processes, and as it is evidenced by the measured PFR curves, the initially optical oriented free excitons can transfer their spin orientation successively to bound excitons and to localized carriers. We have developed in SI a simple two-level model that we can apply to spin oriented excitons bound to donors or to acceptors (the excited state- denoted $\mathrm{D}^{\circ} \mathrm{X}$ or $\mathrm{A}^{\circ} \mathrm{X}$ respectively in section 4 of $\mathrm{SI}$ ), and electron or hole bound to donors or acceptors respectively (the fundamental state- denoted $\mathrm{D}^{\circ}$ or $\mathrm{A}^{\circ}$ respectively in section 4 of SI). This model predicts also the magnetic-field dependence of the 
amplitudes of the excited and fundamental states (see section 4 of the SI). Based on our PFR measurement in absence of a magnetic field (see Figure S4-IIa)), we assume that the spin polarization of the excited states disappears in a very short time $(\leq 100 \mathrm{ps})$ compared to the fundamental state. In a PFR signal, we then mainly observed the electron or hole bound to donors or acceptors respectively (see SI for complementary discussion). In PFR experiments we are always probing a distribution of bound excitons and localized carrriers. At a given laser energy each of them contributes, but the weight are different between them. That is why the ratio between the electron and hole amplitudes varies across the lowest optical transition of MAPI sample (see SI for complementary discussion).

Table 1 summarizes the relaxation times and Landé factors of the electron and hole measured in this work.

\begin{tabular}{ccccc}
\hline \hline Excitation energy $(\mathrm{eV})$ & $T_{2, e}(0)(\mathrm{ns})$ & $T_{2, h}(0)(\mathrm{ns})$ & $\left|g_{e}^{\perp}\right|$ & $\left|g_{h}^{\perp}\right|$ \\
\hline \hline 1.635 & 4.4 & 3.7 & $2.593+/-0.006$ & $0.238+/-0.002$ \\
1.612 & 7 & 3.4 & $2.723+/-0.003$ & $0.350+/-0.003$ \\
\hline \hline
\end{tabular}

Table 1. Relaxation times and transversal Landé factors of the electron and hole obtained at different energies on MAPI sample.

Finally, we have studied the influence of the pump excitation density on the measured coherent evolution of the electronic spin for the two identified species at $1.635 \mathrm{eV}$, the maximum of the lowest-energy absorption band. Fig. 3(a) shows the PFR signal obtained with different pump mean intensities varying from $500 \mu \mathrm{W}$ to $8 \mathrm{~mW}$ (the focus spot beam is $8 \times 10^{-5} \mathrm{~cm}^{2}$ ), at a fixed magnetic field $\mathrm{B}=200 \mathrm{mT}$. The PFR signal obtained at higher pump intensities can be described by the next expression containing a third contribution with amplitude $A_{X}$, dephasing time $T_{2, X}^{*}$ and frequency $f_{X}$ :

$\theta_{F}=A_{e} e^{-t / T_{2, e}^{*}} \cos \left(2 \pi f_{e} t+\varphi_{e}\right)+A_{h} e^{-t / T_{2, h}^{*}} \cos \left(2 \pi f_{h} t+\varphi_{h}\right)+A_{X} e^{-t / T_{2, X}^{*}} \cos \left(2 \pi f_{X} t\right)$ 

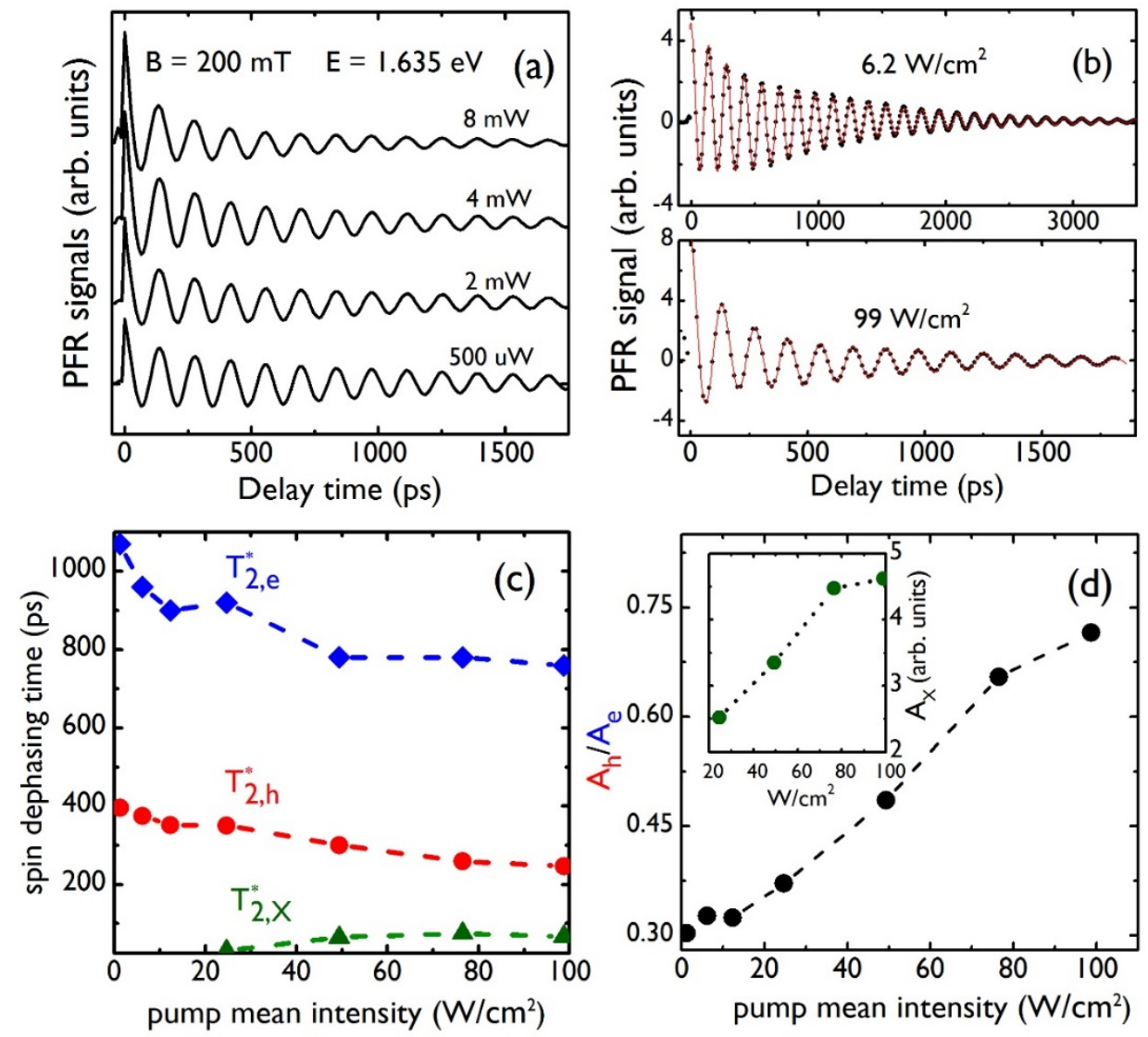

Fig. 3. (a) PFR signals obtained in the magnetic field $B=200 \mathrm{mT}$ using different pump mean powers, at the peak energy of the absorption spectrum $\mathrm{E}=1.635 \mathrm{eV}$. (b) top: PFR signal obtained at a pump mean intensity $6.2 \mathrm{~W} / \mathrm{cm}^{2}$ (black points) and fit to the data (red solid line) with Eq. (1). The focus spot area is $8 \times 10^{-5} \mathrm{~cm}^{2}$. (b) bottom: PFR signal obtained with a pump mean intensity of $99 \mathrm{~W} / \mathrm{cm}^{2}$ and fit (red solid line) through Eq. (4). (c) Spin dephasing times of different contributions to the PFR signal, obtained from the fit of the experimental data through Eq. (1) or Eq. (4), versus pump intensity (d) Evolution of the ratio $A_{h} / A_{e}$, as a function of the pump mean intensities. Inset: evolution of the $A_{X}$ amplitude vs pump mean intensity.

This third contribution appears at $2 \mathrm{~mW}$ and its amplitude increases with the excitation density (see Fig. 3 (d), inset). $T_{2, X}^{*}$ is a very short time of the order of tens of ps and we cannot estimate precisely the third frequency. We assign this third component to the exciton, whose imprint becomes visible when all defects and impurities are saturated at high excitation density. 
Figure 3 (c) shows that, as the pump mean intensity is increased, the spin dephasing times of electron and hole spins slightly shorten. This behavior occurs in II-VI and III-V semiconductors, when high photo-excited carrier concentrations shorten the spin relaxation time due to different mechanisms [45]. Figure 3(d) represents the ratio of the hole amplitude to the electron one. This ratio increases with the density of excitation, whereas it should remain constant if these two components were associated with the fine structure of the exciton.

We placed our study, and previous studies in MAPI, in the general context of the spin physics in more conventional semiconductor materials, and conclude that experimental results cannot be attributed to the beatings of excitons and are more consistent with the localization of electrons and holes. We have demonstrated that PFR experiments in picosecond range is an outstanding tool to explore spin dynamics in hybrid perovskite materials, revealing clear spectroscopic signatures of localized electrons and holes. We have particularly confirmed that, despite a strong spin-orbit coupling, both conduction and valence carriers have long spin lifetime in the range 3-7 ns, making them suitable to spintronic applications.

\section{ASSOCIATED CONTENT}

Supporting Information. Absorption spectrum of the MAPI sample at different temperatures in the range $11 \mathrm{~K}-293 \mathrm{~K}$. Fast Fourier transform of the PFR signals at different magnetic fields obtained at $\mathrm{E}=1.612 \mathrm{eV}$ and $\mathrm{E}=1.635 \mathrm{eV}$. Theoretical calculations for the exciton fine structure in MAPI. Evolution of the PFR amplitudes vs magnetic field obtained at the peak of the absorption spectrum at $2 \mathrm{~K}(\mathrm{E}=1.635 \mathrm{eV})$ and theoretical model. Experimental methods. 
AUTHOR INFORMATION

Corresponding Authors:

Guadalupe Garcia-Arellano - Sorbonne Université, Institut des NanoSciences de Paris, INSP, CNRS-UMR 7588, F-75005 Paris, France

Email : arellano@insp.jussieu.fr

Maria Chamarro - Sorbonne Université, Institut des NanoSciences de Paris, INSP, CNRS-

UMR 7588, F-75005 Paris, France

Email : Maria.Chamarro@insp.jussieu.fr

\section{Authors}

Gaëlle Trippé-Allard - Université Paris-Saclay, ENS Paris-Saclay, Centrale Supélec, CNRS, LuMIn, F-91190 Gif-sur-Yvette, France

Laurent Legrand - Sorbonne Université, Institut des NanoSciences de Paris, INSP, CNRS-

UMR 7588, F-75005 Paris, France

Thierry Barisien- Sorbonne Université, Institut des NanoSciences de Paris, INSP, CNRS-UMR 7588, F-75005 Paris, France

Damien Garrot - Université Paris-Saclay, UVSQ, CNRS, GEMaC, F-78000 Versailles, France

Emmanuelle Deleporte - Université Paris-Saclay, ENS Paris-Saclay, Centrale Supélec, CNRS, LuMIn, F-91190 Gif-sur-Yvette, France

Frédérick Bernardot - Sorbonne Université, Institut des NanoSciences de Paris, INSP, CNRS-

UMR 7588, F-75005 Paris, France

Christophe Testelin- Sorbonne Université, Institut des NanoSciences de Paris, INSP, CNRS-

UMR 7588, F-75005 Paris, France

\section{Notes}

The authors declare no competing financial interests. 


\section{ACKNOWLEDGMENT}

This work was financially supported by the French National Research Agency (ANR IPERNano2, ANR-18-CE30-0023-01 and ANR HYPERSOL, ANR-18-CE05-0021).

\section{REFERENCES}

[1] Miyata, A.; Mitioglu, A.; Plochocka, P.; Portugall, O.; Tse-Wei Wang, J.; Stranks, S. D.; Snaith H. J.; Nicholas, R. J. Direct measurement of the exciton binding energy and effective masses for charge carriers in organic-inorganic tri-halide perovskites, Nature Phys. 2015, 11, $582-588$.

[2] Galkowski, K.; Mitioglu, A.; Miyata, A.; Plochocka, P.; Portugall, O.; Eperon, G. E.; TseWei Wang, J.; Stergiopoulos, T.; Stranks, S. D.; Snaithc, H. J.; Nicholas, R. J. Determination of the exciton binding energy and effective masses for methylamonium and formamidinium lead tri-halide perovskite semiconductors, Energy Environ. Sci. 2016, 9, 962-970.

[3] Baranowski, M. and Plochocka, P. Excitons in metal-halide perovskites, Adv. Energy Mater. 2020, 10, 1903659.

[4] Baranowski, M.; Plochocka, P.; Su, R.; Legrand, L.; Barisien, T.; Bernardot, F.; Xiong, Q.; Testelin, C.; Chamarro, M. Exciton binding energy and effective mass of $\mathrm{CsPBCl}_{3}$ : a magnetooptical study, Photonics Res. 2020, 8 (10), A50-A55.

[5] Weisbuch, C.; Benisty, H.; Houdré, R.; Overview of fundamentals and applications of electrons and photons in confined structures, J. of Lum 2000, 85, 271-293.

[6] Horodyski, P. and Hildek, P. Free-exciton absorption in bulk CdTe absorption: temperature dependence, Physica Status Solidi B 2006, 243, 494-501.

[7] Halliday, D. P.; Potter, M. D. G.; Mullins, J. T.; Brinkman, A.W. Photoluminescence study of a bulk vapor grown CdTe crystal, J. of Crystal Growth 2000, 220, 30-38.

[8] Fang, H.-H.; Raissa, R.; Abdu-Aguye, M.; Adjokatse, S.; Blake, G. R.; Even, J.; Loi, M. A. Photophysics of organic-inorganic hybrid lead iodide perovskite single crystals, Adv. Funct. Mater. 2015, 25, 2378-2385.

[9] Phuong, L. Q.; Yamada, Y.; Nagai, M.; Maruyama, N.; Wakamiya, A.; Kanemitsu, Y. Free Carriers versus excitons in $\mathrm{CH}_{3} \mathrm{NH}_{3} \mathrm{PbI}_{3}$ perovskite thin films at low temperatures: charge transfer from the orthorhombic phase to the tetragonal phase, J. Phys. Chem. Letters, 2016, 7, 2316-2016. 
[10] Plantevin, O.; Valère, S.; Guerfa, D.; Lédée, F.; Trippé-Allard, G.; Garrot, D. and Deleporte, E. Photoluminescence Tuning Through Irradiation Defects in $\mathrm{CH}_{3} \mathrm{NH}_{3} \mathrm{PbI}_{3}$ Perovskites, Physica Status Solidi 2019, 256, 1900199.

[11] Francisco-López, A; Charles, B.; Alonso, M.-I.; Garriga, M.; Weller, M. T. and Goñi A. R. Photoluminescence of Bound-Exciton Complexes and Assignment to Shallow Defects in Methylammonium/Formamidinium Lead Iodide Mixed Crystals, Adv. Optical. Mater. 2021, 2001969.

[12] Yin W. J.; Shin T.; and Yan Y. Unusual defect physics in $\mathrm{CH}_{3} \mathrm{NH}_{3} \mathrm{PbI}_{3}$ perovskite solar cell absorber, Appl. Phys. Lett. 2014, 104, 063903.

[13] Kim J.; Lee S.-H.; Lee J. H.; Hong K. H. The Role of Intrinsic Defects in Methylammonium Lead Iodide Perovskite, J. Phys. Chem. Lett. 2014, 5, 8, 1312-1317.

[14] Kepenekian, M.; Robles, R.; Katan, C.; Sapori, D.; Pedesseau, L.; Even, J. Rashba and Dresselhaus effects in hybrid organic-inorganic perovskites: from basics to devices, ACS Nano 2015, 9, 11557-11567.

[15] Kepenekian, M.; Even, J. Rashba and Dresselhaus couplings in Halide perovskites: Accomplishments and opportunities for spintronics and spin-orbitronics, J. Phys. Chem. Lett. 2017, 8, 14, 3362-3370.

[16] Odenthal, P.; Talmadge, W.; Gundlach, N.; Wang, R.; Zhang, C.; Sun, D.; Yu, Z.- G.; Vardeny, Z. V.; Li, Y. S. Spin-polarized exciton quantum beating in hybrid organic-inorganic perovskites, Nat. Phys. 2017, 13, 894-899.

[17] Belykh, V. V.; Yakovlev, D Y.; Glazov, M. M.; Grigoryev, P. S.; Hussain, M.; Rautert, J.; Dirin, D. N.; Kovalenko, M. V. and Bayer, M. Coherent spin dynamics of electrons and holes in $\mathrm{CsPbBr}_{3}$ perovskite crystals, Nat. Commun. 2019, 10, 673.

[18] Giovanni, D.; Ma, H.; Chua, J.; Gratzel, M.; Ramesh, R.; Mhaisalkar, S.; Mathews, N.; Sum, T. Z. Highly Spin-Polarized Carrier Dynamics and Ultralarge Photoinduced Magnetization in $\mathrm{CH}_{3} \mathrm{NH}_{3} \mathrm{PbI}_{3}$ Perovskite Thin Films, Nano Lett. 2015, 15, 1553-1558.

[19] Zhou, M. ; Sarmiento, J. S., Fei, C. ; Zhang, X. and Zhou, H. W. Effect of the composition on the spin relaxation of Lead halide perovskites, J. Phys. Chem. Lett. 2020, 11, 1502-1507.

[20] Schuster, O.; Wientjes, P.; Shrestha, S.; Levchuk, I.; Sytnyk, M.; Matt, G. J.; Osvet, A; Batentschuk, M.; Heiss, W.; Brabec, C. J.; Fauster, T.; Niesner, D. Looking beyond the Surface: The Band Gap of Bulk Methylammonium Lead Iodide, Nano Lett. 2020, 20, 5, 3090-3097. 
[21] Chamarro, M.; Bernardot, F.; and Testelin, C.; Spin decoherence and relaxation processses in zero-dimensional semiconductor nanostructures, J. of Phys.: Condens. Matter 2007, 19, 445007.

[22] Diab, H.; Trippé-Allard, G.; Ledée, F.; Jemli, K.; Vilar, C.; Bouchez, G.; Jacques, V. L. R.; Tejeda, A.; Even, J.; Lauret, J.-S.; Deleporte, E.; and Garrot, G. Narrow Linewidth Excitonic Emission in Organic-Inorganic Lead Iodide Perovskite Single Crystals. J. Phys. Chem. Lett. 2016, 7, 24, 5093-5100.

[23] Ramade, J.; Andriambariarijaona, L. M.; Steinmetz, V.; Goubet, N.; Legrand, L.; Barisien, T.; Bernardot, F.; Testelin, C.; Lhuillier, E.; Bramati, A.; Chamarro, M.; Fine structure of excitons and electron-hole exchange energy in polymorphic $\mathrm{CsPBr}_{3}$ single nanocrystals, Nanoscale 2018, 10, 6393-6401.

[24] Ben Aich, R.; Saïdi, I.; Ben Radhia, S.; Boujdaria, K.; Barisien, T.; Legrand, L.; Bernardot, F.; Chamarro, M.; Testelin, C. Bright- Exciton Splittings in Inorganic Cesium Lead Halide Perovskite Nanocrystals. Phys. Rev. Appl. 2019, 11, 034042.

[25] Ben Aich, R.; Radhia, S. B.; Boujdaria, K.; Chamarro, M.; Testelin, C.; Multiband k.p Model for Tetragonal Crystals: Application to Hybrid Halide Perovskite Nanocrystals, J. Phys. Chem. Lett. 2020, 11, 3, 808-817.

[26] Sercel, P. C; Lyons, J. L.; Bernstein, N.; and Efros, A. L. Quasicubic model for metal halide perovskite nanocrystals, J. Chem. Phys. 2019, 151, 234106.

[27] Baranowski, M.; Galkowski, K.; Surrente, A.; Urban, J.; Kłopotowski, Ł.; Macḱowski, S.; Maude, D. K.; Aich, R. B.; Boujdaria, K.; Chamarro, M.; Testelin, C.; Nayak, P. K.; Dollmann, M.; Snaith, H. J.; Nicholas, R. J.; Plochocka, P.; Giant Fine Structure Splitting of the Bright Exciton in a Bulk MAPbBr 3 Single Crystal, Nano Lett. 2019, 19, 10, 7054-7061.

[28] Kikkawa, J. M.; Awschalom, D. D. Resonant Spin Amplification in n-Type GaAs, Phys. Rev. Lett. 1998, 80, 4313.

[29] Dzhioev, R. I.; Kavokin, K. V.; Korenev, V. L.; Lazarev, M. V.; Meltser, B. Y.; Stepanova, M. N.; Zakharchenya, B. P.; Gammon, D.; Katzer, D. S. Low-temperature spin relaxation in $n$ type GaAs, Phys. Rev. B 2002, 66, 245204.

[30] Sprinzl, D.; Horodyská, P.; Tesařová, N.; Rozkotová, E.; Belas, E.; Grill, R.; Malý, P.; Němec, P. Influence of n-type doping on electron spin dephasing in CdTe, Phys. Rev. B 2010, 82, 153201. 
[31] Yu, Z. Effective-mass model and magneto-optical properties in hybrid perovskites. Sci Rep 2016, 6, 28576.

[32] Amand, T. ; Marie, X. ; Le Jeune, P. ; Brousseau, M. ; Robart, D. ; Barrau, J. ; Planel, R. Spin Quantum Beats of 2D Excitons, Phys. Rev. Lett. 1997, 78, 1355.

[33] Bar-Ad, S.; Bar-Joseph, I. Absorption Quantum Beats of Magnetoexcitons in GaAs Heterostructures, Phys. Rev. Lett. 1991, 66, 2491; Exciton Spin Dynamics in GaAs Heterostructures, Phys. Rev. Lett. 1992, 68, 349.

[34] Lenihan, A. S.; Gurudev Dutt, M. V.; Steel, D. G.; Ghosh, S.; Bhattacharya, P. K. Raman Coherence Beats from Entangled Polarization Eigenstates in InAs Quantum Dots, Phys. Rev. Lett. 2002, 88, 223601.

[35] Sénès, M.; Urbaszek, B.; Marie, X.; Amand, T.; Tribollet, J.; Bernardot, F.; Testelin, C.; Chamarro, M.; Gérard, J.-M.; Exciton spin manipulation in InAs/GaAs quantum dots: Exchange interaction and magnetic field effects, Phys. Rev. B 2005, 71, 115334.

[36] Bernardot, F.; Aubry, E.; Tribollet, J.; Testelin, C.; Chamarro, M.; Lombez, L.; Braun, P.F.; Marie, X.; Amand, T.; Gérard, J.-M. Linear and dynamical photoinduced dichroisms of InAs/GaAs self-assembled quantum dots: Population relaxation and decoherence measurements, Phys. Rev. B 2006, 73, 085301.

[37] Testelin, C.; Aubry, E.; Chaouache, M.; Maaref, M.; Bernardot, F.; Chamarro, M. and Gérard, J.-M. Energy dependence of the electron-hole in-plane anisotropy in InAs/GaAs quantum dots, Phys. Stat. Sol. (c) 2006, 3, 3900-3903.

[38] Rainò, G.; Nedelcu, G.; Protesescu, L.; Bodnarchuk, M.I.; Kovalenko, M. V.; Mahrt, R. F.; Stöferle, T. Single cesium lead halide perovskite nanocrystals at low temperature: Fast SinglePhoton Emission, Reduced Blinking, and Exciton Fine Structure, ACS Nano 2016, 10, 2, 24852490 .

[39] Fu, M.; Tamarat, P.; Huang, H.; Even, J.; Rogach, A. L.; Lounis, B. Neutral and Charged Exciton Fine Structure in Single Lead Halide Perovskite Nanocrystals Revealed by Magnetooptical Spectroscopy, Nano Lett. 2017, 17, 5, 2895-2901.

[40] Tamarat, P.; Bodnarchuk, M. I.; Trebbia, J.-P.; Erni, R.; Kovalenko, M. V.; Even, J.; Lounis, B. The Ground Exciton State of Formamidinium Lead Bromide Perovskite Nanocrystals is a Singlet Dark. Nat. Mater. 2019, 18, 717-724. 
[41] Tamarat, P.; Hou, L.; Trebbia, J.-B.; Swarnkar, A.; Biadala, L.; Louyer, Y.; Bodnarchuk, M. I.; Kovalenko, M. V.; Even, J.; and Lounis, B. The dark exciton ground state promotes photon-pair emission in individual perovskite nanocrystals. Nat Commun 11, 6001 (2020).

[42] Merkulov, I. A.; Efros, Al. L.; Rosen, M.; Electron spin relaxation by nuclei in semiconductor quantum dots, Phys. Rev. B 2002, 65, 205309.I. A. Merkulov, Al. L. Efros, and M. Rosen, Phys. Rev. B 65, 205309 (2002).

[43] Kavokin, K.V. Anisotropic exchange interaction of localized conduction-band electrons in semiconductors, Phys. Rev. B 2001, 64, 075305.

[44] Lonnemann, J. G.; Rugeramigabo, E. P.; Oestreich, M.; Hübner, J. Closing the gap between spatial and spin dynamics of electrons at the metal-to-insulator transition, Phys. Rev. B 2017, 96, 045201.

[45] Garcia-Arellano, G.; Bernardot, F.; Karczewski, G.; Testelin, C.; and Chamarro, M.; Spin relaxation time of donors-bound electrons in a CdTe quantum well, Phys. Rev. B 2019, 99, 235301 